\title{
COMPUTER DYNAMICS SIMULATION OF DRUG DEPENDENCE THROUGH ARTIFICIAL NEURONAL NETWORK: PEDAGOGICAL AND CLINICAL IMPLICATIONS
}

\author{
SANTOS, ${ }^{1}$. ; TORRES, B.B..$^{2}$; GALEMBECK, E. ${ }^{1}$ \\ ${ }^{1}$ Departamento de Bioquímica, Universidade Estadual de Campinas; \\ ${ }^{2}$ Departamento de Bioquímica, Instituto de Química, Universidade de São \\ Paulo.
}

To develop and to evaluate the efficiency of a software able to simulate a virtual patient at different stages of addition was the main goal and challenge of this work. We developed the software in Borland ${ }^{\mathrm{TM}}$ Delphi $5^{\circledR}$ programming language. Techniques of artificial intelligence, neuronal networks and expert systems, were responsible for modeling the neurobiological structures and mechanisms of the interaction with the drugs used. Dynamical simulation and hypermedia were designed to increase the software's interactivity which was able to show graphical information from virtual instrumentation and from realistic functional magnetic resonance imaging display. Early, the program was designed to be used by undergraduate students to improve their neurophysiologic learn, based not only in an interaction of membrane receptors with drugs, but in such a large behavioral simulation. The experimental manipulation of the software was accomplished by: i) creating a virtual patient from a normal mood to a behavioral addiction, increasing gradatively: alcohol, opiate or cocaine doses. ii) designing an approach to treat the patient, to get total or partial remission of behavioral disorder by combining psychopharmacology and psychotherapy. Integration of dynamic simulation with hypermedia and artificial intelligence has been able to point out behavioral details as tolerance, sensitization and level of addiction to drugs of abuse and so on, turned into a potentially useful tool in the development of teaching activities on several ways, such as education as well clinical skills, in which it could assist patients, families and health care to improve and test their knowledge and skills about different faces supported by drugs dependency. Those features are currently under investigation. 